\title{
Causal Structure of Relativistic Dissipative Hydrodynamics
}

\author{
G. S. Denicol, Ph. Mota, T. Kodama, and T. Koide \\ Instituto de Física, Universidade Federal do Rio de Janeiro, C.P. 68528, 21945-970 Rio de Janeiro, RJ, Brazil
}

Received on 29 September, 2006

\begin{abstract}
We present a new formalism for the relativistic dissipative hydrodynamics consistent with causality. We start from the physical analysis of the irreversible currents according to the Landau-Lifshitz theory. Then, the irreversible currents are given by integral expressions which take into account the relaxation time. Only one additional parameter was introduced, the relaxation time, $\tau_{R}$. We verified that the linearized equation of motion for small perturbations in the homogeneous, static background coincides with Hiscock-Lindblom
\end{abstract}

Keywords: Viscosity; Dissipative phenomena; Relativistic hydrodynamics

\section{INTRODUCTION}

The ideal hydrodynamical description for the dynamics of hot and dense matter achieved in the RHIC experiments works amazingly well, particularly for the behavior of collective flow parameters. However, we know that there still exist several open problems in the interpretation of data in terms of the hydrodynamical model [1]. These questions require careful examination to extract quantitative and precise information on the properties of QGP. In particular, we should study the effect of dissipative processes on the collective flow variables. Several works have been done in this direction [2]. However, strictly speaking, a quantitative and consistent analysis of the viscosity within the framework of relativistic hydrodynamics has not yet been done completely. This is because the introduction of dissipative phenomena in relativistic hydrodynamics casts difficult problems, both conceptual and technical. Initially Eckart, and later, Landau-Lifshitz introduced the dissipative effects in relativistic hydrodynamics in a covariant manner [3, 4]. It is, however, known that their formalism leads to the problem of acausality, that is, a pulse signal propagates with infinite speed. Thus, relativistic covariance is not a sufficient condition for a consistent relativistic dissipative dynamics $[5,6]$.

\section{PROBLEM OF CAUSALITY IN DIFFUSION PROCESS}

The fundamental problem of the first order theory like the Navier-Stokes theory is attributed to the fact that the diffusion equation is parabolic. The diffusion process is a typical relaxation process of conserved quantities. Thus, it should satisfy the equation of continuity,

$$
\frac{\partial n}{\partial t}+\nabla \cdot \vec{j}=0
$$

where $n$ is a density of a conserved quantity. The irreversible current $\vec{j}$ is, phenomenologically assumed to be proportional to a thermodynamic force $F$, and it is phenomenologically given by the gradient of $n$,

$$
\vec{j}=-\zeta \vec{F}=-\zeta \nabla n
$$

where $\zeta$ is the Onsager coefficient. Substituting Eq.(2) into Eq.(1), we get the diffusion equation,

$$
\frac{\partial}{\partial t} n=\zeta \nabla^{2} n
$$

Fick's law tells us that the above diffusion process is induced by an inhomogeneous distribution. In Eq.(2), the irreversible current is directly proportional to the space inhomogeneity. However, this is a very special case. In general, the generation of a irreversible current has a time delay. Thus, we may think of memory effects within the linear response of the system. Phenomenologically, this can be done by introducing the following memory function $[7,8]$,

$$
\begin{aligned}
G\left(t, t^{\prime}\right) & =\frac{1}{\tau_{R}} e^{-\left(t-t^{\prime}\right) / \tau_{R}}, t \geq t^{\prime} \\
& =0, \quad t<t^{\prime}
\end{aligned}
$$

where $\tau_{R}$ is a constant (relaxation time) and rewrite Eq.(2) as

$$
\vec{j}=-\int_{-\infty}^{t} G\left(t, t^{\prime}\right) \zeta \vec{F}\left(t^{\prime}\right) d t^{\prime}
$$

In the limit of $\tau_{R} \rightarrow 0$, we have $G\left(t, t^{\prime}\right) \rightarrow \delta\left(t-t^{\prime}\right)$ so that the original equation (2) is recovered [9]. Substituting into the equation of continuity (1), we arrive at

$$
\frac{\partial^{2} n}{\partial t^{2}}=\frac{1}{\tau_{R}}\left(-\frac{\partial n}{\partial t}+\zeta \nabla^{2} n\right) .
$$

This equation is hyperbolic. This telegraph equation is sometimes called the causal diffusion equation.

For a suitable choice of the parameters $\tau_{R}$ and $\zeta$, we can recover the causal propagation of diffusion process. In fact, the maximum velocity of the signal propagation governed by this equation is shown to be [10]

$$
v_{\max }=\sqrt{\frac{\zeta}{\tau_{R}}} .
$$

The diffusion equation corresponds to $\tau_{R}=0$ and hence $v_{\max } \rightarrow \infty$. This is the reason why the diffusion equation breaks causality. 


\section{RELATIVISTIC DISSIPATIVE HYDRODYNAMICS}

Eckart and Landau-Lifshitz derived the relativistic dissipative hydrodynamics following non-equilibrium thermodynamics as discussed in the preceding section [3, 4]. Their theories are just the covariant versions of the Navier-Stokes equation and the corresponding equations still continue to be parabolic.

As a matter of fact, the irreversible currents of the LandauLifshitz theory (LL) are constructed as follows. First of all, the energy-momentum tensor is expressed as

$$
T^{\mu \nu}=\varepsilon u^{\mu} u^{v}-P^{\mu \nu}(p+\Pi)+\pi^{\mu \nu},
$$

where, $\varepsilon, u^{\mu}, \Pi$ and $\pi^{\mu \nu}$ are respectively the energy density, the four velocity of the fluid and the bulk and shear viscous stresses. In the LL, the velocity field is defined in such a way that the energy current vanishes in the local rest frame, $u^{\mu} \rightarrow(1,0,0,0)$. In this local rest frame, it is assumed that the equation of state and thermodynamical relations are valid as if it were in equilibrium. As usual, we write

$$
u^{\mu}=\left(\begin{array}{c}
\gamma \\
\gamma \vec{v}
\end{array}\right)
$$

where $\gamma$ is the Lorentz factor and

$$
u^{\mu} u_{\mu}=1
$$

The tensor $P^{\mu \nu}$ is the projection operator to the space orthogonal to $u^{\mu}$ and given by

$$
P^{\mu v}=g^{\mu v}-u^{\mu} u^{v} .
$$

On the other hand, the current for the conserved quantity (e.g., baryon number) takes the form

$$
N^{\mu} u^{\mu}+v^{\mu}
$$

where $v^{\mu}$ is the heat conduction part of the current.

It should be noted that for the irreversible currents, we require the constraints [4],

$$
u_{\mu} \pi^{\mu v}=0
$$

and

$$
u_{\mu} v^{\mu}=0 .
$$

Then, the divergence of the entropy four flux is given by

$$
\partial_{\mu}\left(s u^{\mu}-\alpha v^{\mu}\right)=\frac{1}{T}\left(-P^{\mu v} \Pi+\pi^{\mu v}\right) \partial_{\mu} u_{v}-v^{\mu} \partial_{\mu} \alpha,
$$

where $\alpha=\mu / T$ and $\mu$ is the chemical potential. Due to the second law of thermodynamics, the r.h.s. of the equation should be positive. Then, the irreversible currents are given by

$$
\begin{aligned}
\Pi & =-\zeta F=-\zeta \partial_{\alpha} u^{\alpha}, \\
\pi_{\mu v} & =P_{\mu v \alpha \beta} \widetilde{\pi}^{\alpha \beta}=\eta P_{\mu v \alpha \beta} F^{\alpha \beta}=\eta P_{\mu v \alpha \beta} \partial^{\alpha} u^{\beta}, \\
v_{\mu} & =P_{\mu \nu} \widetilde{v}^{v}=-\kappa P_{\mu \nu} F^{v}=-\kappa P_{\mu v} \partial^{v} \alpha,
\end{aligned}
$$

where $\zeta, \eta$ and $\kappa$ are bulk viscosity, shear viscosity and thermal conductivity coefficients, respectively. Here, $P^{\mu \alpha v \beta}$ is the double symmetric traceless projection operator,

$$
P^{\mu \nu \alpha \beta}=\frac{1}{2}\left(P^{\mu \alpha} P^{v \beta}+P^{\mu \beta} P^{v \alpha}\right)-\frac{1}{P_{\lambda}^{\lambda}} P^{\mu v} P^{\alpha \beta},
$$

and we have introduced the quantities $\widetilde{\pi}^{\alpha \beta}$ and $\widetilde{v}^{v}$ which correspond respectively to the shear tensor and irreversible current before the projection. They are in general not orthogonal to $u^{\mu}$ so the projection operators are necessary to satisfy the constraints Eqs.(7) and (8).

One can see that the irreversible currents are induced by inhomogeneous distribution, and the space inhomogeneity immediately gives rise to the irreversible current. This is the same structure as the diffusion equation. In this sense, the LL is parabolic and does not obey causality. To solve this problem, we will introduce the memory effect in the same way as in the diffusion equation. Then, we use the same memory function as Eq. (3). Thus, the modified irreversible currents are

$$
\begin{aligned}
\Pi(\tau) & =-\int_{-\infty}^{\tau} d \tau^{\prime} G\left(\tau, \tau^{\prime}\right) \zeta \partial_{\alpha} u^{\alpha}\left(\tau^{\prime}\right), \\
\tilde{\pi}^{\mu \nu}(\tau) & =\int_{-\infty}^{\tau} d \tau^{\prime} G\left(\tau, \tau^{\prime}\right) \eta \partial^{\mu} u^{\nu}\left(\tau^{\prime}\right), \\
\widetilde{v}^{\mu}(\tau) & =-\int_{-\infty}^{\tau} d \tau^{\prime} G\left(\tau, \tau^{\prime}\right) \kappa \partial^{\mu} \alpha\left(\tau^{\prime}\right),
\end{aligned}
$$

where $\tau=\tau(\vec{r}, t)$ is the local proper time. As before, the shear tensor $\pi^{\mu \nu}$ and the irreversible current $\nu^{\mu}$ are given by the projection of these integrals as

$$
\begin{aligned}
\pi^{\mu v} & =P^{\mu v \alpha \beta} \tilde{\pi}_{\alpha \beta}(\tau), \\
v^{\mu} & =P^{\mu v} \widetilde{v}_{v}(\tau) .
\end{aligned}
$$

It is important to remark that in the limit of $\tau_{R} \rightarrow 0$, we have $G\left(t, t^{\prime}\right) \rightarrow \delta\left(t-t^{\prime}\right)$ so that the original equations (10) are recovered. When we start with the finite initial time, say $\tau_{0}$, the above integrals should read

$$
\begin{gathered}
\Pi(\tau)=-\int_{\tau_{0}}^{\tau} d \tau^{\prime} G\left(\tau, \tau^{\prime}\right) \zeta \partial_{\alpha} u^{\alpha}\left(\tau^{\prime}\right)+e^{-\left(\tau-\tau_{0}\right) / \tau_{R}} \Pi_{0}, \\
\tilde{\pi}^{\mu v}(\tau)=\int_{\tau_{0}}^{\tau} d \tau^{\prime} G\left(\tau, \tau^{\prime}\right) \eta \partial^{\mu} u^{\nu}\left(\tau^{\prime}\right)+e^{-\left(\tau-\tau_{0}\right) / \tau_{R}} \tilde{\pi}_{0}^{\mu v}, \\
\widetilde{v}^{\mu}(\tau)=-\int_{\tau_{0}}^{\tau} d \tau^{\prime} G\left(\tau, \tau^{\prime}\right) \kappa \partial^{\mu} \alpha\left(\tau^{\prime}\right)+e^{-\left(\tau-\tau_{0}\right) / \tau_{R} \widetilde{v}_{0}^{\mu}}
\end{gathered}
$$

Till now, we have considered that the relaxation time $\tau_{R}$ is constant. However in practical problems, it is a function of thermodynamical variables. Then the memory function should be generalized as

$$
G\left(\tau, \tau^{\prime}\right) \rightarrow \frac{1}{\tau_{R}\left(\tau^{\prime}\right)} e^{-\int_{\tau^{\prime}}^{\tau} \frac{1}{\tau_{R}\left(\tau^{\prime \prime}\right)} d \tau^{\prime \prime}} .
$$

In our formulation, the irreversible currents are modified by introducing the memory function. However, the original currents are defined to satisfy the second law of thermodynamics. Therefore, our definition, in general, does not satisfy it. For example, if the relaxation time $\tau_{R}$ becomes large, the entropy production becomes negative [11]. 


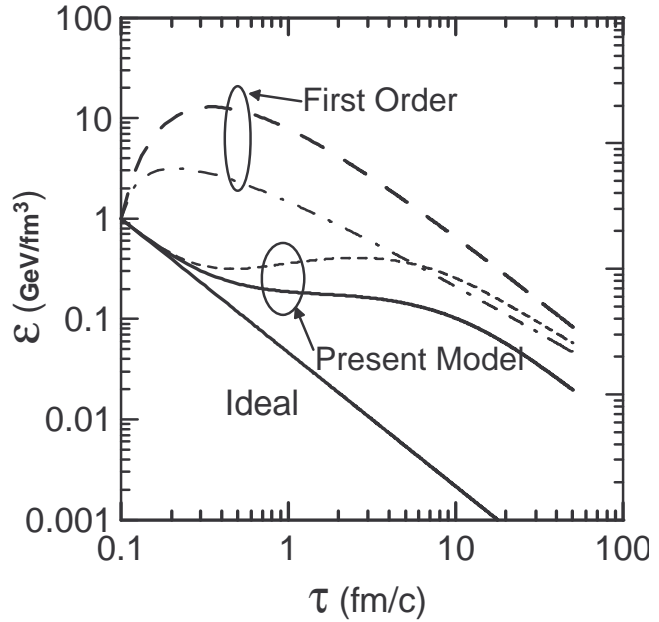

FIG. 1: The time evolution of the energy density. The dashed curves correspond to the calculations with the constant viscosity and relaxation time. The first two lines from the top represents the results of the LL. Next two lines shows the results of our theory. The last line is the result of ideal hydrodynamics.

\section{BJORKEN'S SCALING SOLUTION}

To see how the above scheme works, let us apply it to the one dimensional scaling solution of the Bjorken model.

The time component of the divergence of $T^{\mu v}$ gives

$$
\frac{d}{d \tau} \varepsilon(\tau)+\frac{\varepsilon+P+\Pi}{\tau}+\frac{2}{3} \frac{\Omega}{\tau}=0,
$$

where

$$
\Omega(\tau)=-\int_{\tau_{0}}^{\tau} d \tau^{\prime} G\left(\tau, \tau^{\prime}\right) \frac{\eta}{\tau^{\prime}}+\tau_{R}\left(\tau_{0}\right) G\left(\tau, \tau_{0}\right) \Omega\left(\tau_{0}\right) .
$$

The equation for the space component is automatically satisfied by the scaling ansatz showing its consistency. The entropy production rate is calculated to be

$$
\partial_{\mu}\left(s u^{\mu}-\alpha v^{\mu}\right)=-\frac{1}{T} \frac{1}{\tau}\left(\Pi+\frac{2}{3} \Omega\right),
$$

Since $\Pi$ and $\Omega$ are negative definite, the entropy production is positive definite. For simplicity, we consider only the effect of $\Omega$.

A typical estimate from the kinetic theory shows that the shear viscosity $\eta$ is proportional to the entropy density $s, \eta=$ $b s$, where $b$ is a constant [12,13]. Following Ref. [13], we choose $b=1.1$. Furthermore, we use the relaxation time [12, 13] $\tau_{R}=\frac{3 \eta_{I S}}{4 p}=\frac{3 \eta}{8 p}$. We further assume the equation of state of the ideal pion gas.

In Fig. 1, we show the energy density $\varepsilon$ obtained by solving Eq. (17) as function of proper time $\tau$. As a initial condition, we set $\varepsilon\left(\tau_{0}\right)=1 \mathrm{GeV} / \mathrm{fm}^{3}, \Pi\left(\tau_{0}\right)=\Omega\left(\tau_{0}\right)=0$ at the initial

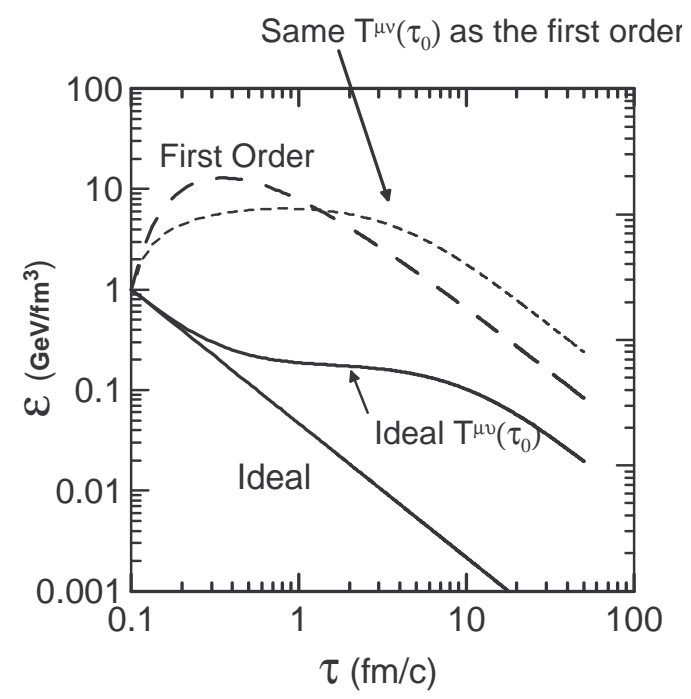

FIG. 2: The time evolution of energy density with the different initial conditions from Fig. 1. The dashed and short dashed lines represent the result of the LL and our theory, respectively. For comparison, our result of Fig. 1 is shown, again (ideal $T^{\mu v}\left(\tau_{0}\right)$ ). The last line from the top is the result of ideal hydrodynamics. In this case, the energy heat-up is observed even in our theory.

proper time $\tau_{0}=0.1 \mathrm{fm} / \mathrm{c}$. The first two lines from the top represents the results of the LL. The next two lines shows the results of our theory. The last line is the result of ideal hydrodynamics. For the solid lines, we calculated with the viscosity and relaxation time which depend on temperature. Initially, the effect of viscosity is small because of the memory effect and the behavior of our theory is similar to that of ideal hydrodynamics. After a time larger than the relaxation time, the memory effect is not effective anymore and the behavior is similar to the result of the LL. As we have mentioned, the behavior of our theory is the same as the result obtained in Ref. [13] in this case. For the dashed lines, we calculated with the constant viscosity and relaxation time, $\eta=\eta\left(\varepsilon_{0}\right)$ and $\tau_{R}=\tau_{R}\left(\varepsilon_{0}\right)$. In this case, the viscosity is constant so that the heat production stays longer and has a smaller slope as function of time asymptotically.

Sometimes the emergence of the initial heat-up in the LL (the dashed curve in Fig. 1) is interpreted as an intrinsic problem of the first order theory. However, such behavior can also appear even in the second order theory. In Fig. 2, we set $\Pi\left(\tau_{0}\right)=\zeta\left(\tau_{0}\right) / \tau_{0}$ and $\Omega\left(\tau_{0}\right)=\eta\left(\tau_{0}\right) / \tau_{0}$ as the initial conditions. In particular, the initial heat-up also appears in the second order depending on the initial condition for the irreversible currents (see Fig. 2). Therefore, this heat-up is not a problem of the first order theory but rather the specific property of the scaling ansatz. This was already pointed out by Muronga. The physical reason for this heat-up is due to the use of the Bjorken solution for the velocity field. In this case, the system acts as if an external force is applied to keep the velocity field as a given function of $\tau$. Thus, depending on the 
relative intensity of the viscous terms compared to the pressure, the external work converted to the local heat production can overcome the temperature decrease due to the expansion.

\section{SUMMARY AND CONCLUDING REMARKS}

In this report, we presented a new formalism for the relativistic dissipative hydrodynamics consistent with causality. We start from the physical analysis of the irreversible currents according to the Landau-Lifshitz theory. Then, the irreversible currents are given by integral expressions which take into account the relaxation time. In this way, causality is recovered and at the same time a simple physical structure of the LL is preserved. In our approach, only one additional parameter was introduced, the relaxation time, $\tau_{R}$. The resulting equation of motion then becomes hyperbolic and causality can be restored [5]. Naturally, causality depends on the choice of the values of the parameters including the relaxation time.

More specifically, we verified that the linearized equation of motion for small perturbations in the homogeneous, static background coincides with Hiscock-Lindblom [14-16] ex- cept for the coupling among the different irreversible currents. These couplings are not included in our theory considering the Curie principle. Of course the Curie principle is believed to be valid in the regime of the first order theory and in the second order regime these couplings might be present. However, the existence of the Curie principle may imply that these couplings are small compared with the direct terms.

We have applied our theory to the case of the onedimensional scaling solution of Bjorken and obtained the analogous behavior of previous analysis. In this case we can prove explicitly the positiveness of entropy production. We showed the time evolution of the temperature. As expected, our theory gives the same result of Ref. [13], because the no-acceleration condition used in Ref. [13] is automatically satisfied in this model. Note that our theory is applicable to more general case where the acceleration is important.

Our theory is particularly adequate to be applied to a hydrocode such as SPheRIO which is based on the Lagrangian coordinate system $[17,18]$. Implementation of the present theory to the full three-dimensional hydrodynamics is now in progress.
[1] T. Kodama, T. Koide, G. S. Denicol, and Ph. Mota, hep$\mathrm{ph} / 0606161$.

[2] D. Teaney, Phys. Rev. C 68, 034913 (2003).

[3] C. Eckart, Phys. Rev. 58, 919 (1940).

[4] L. D. Landau and E. M. Lifshitz, Fluid Mechanics (Pergamon, New York, 1959).

[5] I. Müller, Living Rev. Rel. 2, 1 (1999).

[6] D. Jou, J. Casas-Vázquez, and G. Lebon, Rep. Prog. Phys. 51, 1105 (1988); Rep. Prog. Phys. 62, 1035 (1999),

[7] M. A. Aziz and S. Gavin, Phys. Rev. C 70, 034905 (2004).

[8] T. Koide, G. Krein, and R. O. Ramos, Phys. Lett. B 636, 96 (2006).

[9] Such a memory function was also used in the hydrodynamic equations to take into account the time delay necessary to thermalize the micro turbulences in a large scale system such as supernova explosion. See T.Kodama, R. Donangelo and M. Guidry, Int. J. Mod. Phys. C 9, 745 (1998).
[10] P. M. Morse and H. Feshbach, Methods of Theoretical Physics, (McGraw Hill, New York, 1953), p865.

[11] T. Koide, G. S. Denicol, Ph. Mota, and T. Kodama, hep$\mathrm{ph} / 0909117$.

[12] A. Muronga, Phys. Rev. C 69, 034903 (2004).

[13] R. Baier, P. Romatschke, and U. A. Wiedemann, Phys. Rev. C 73, 064903 (2006).

[14] W. A. Hiscock and L. Lindblom, Ann. Phys. (N.Y.) 151, 466 (1983).

[15] W. A. Hiscock and L. Lindblom, Phys. Rev. D 31, 725 (1985).

[16] W. A. Hiscock and L. Lindblom, Phys. Rev. D 35, 3723 (1987).

[17] C. E. Aguiar, T. Kodama, T. Osada, and Y. Hama, J. Phys. G 27, 75 (2001).

[18] Y. Hama, T. Kodama, and O. Socolowski, Braz. J. Phys. 35, 24 (2005). 